\title{
Hydrogen Atom Transfer Reactions of Imido Manganese(V) Corrole: One Reaction with Two Mechanistic Pathways
}

Michael J. Zdilla, Jennifer L. Dexheimer, and Mahdi M. Abu-Omar

Brown Laboratory, Department of Chemistry, Purdue University, 560 Oval Drive, West Lafayette, IN 47907 


\section{Table of Contents}

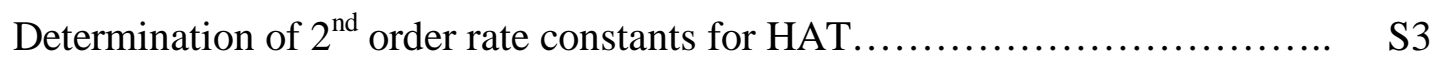

Repeat Scans on Reaction of 2 with 2,6-di-tert-butylphenol................ S5

EPR of phenoxyl radicals ........................................ S6

Activation parameters........................................... S7

Isokinetic correlation plot....................................... S8

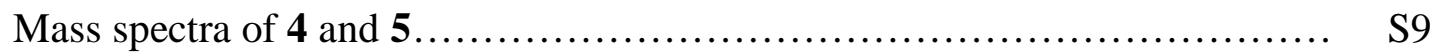




\section{Determination of $2^{\text {nd }}$ order rate constant for hydrogen abstraction by 2}
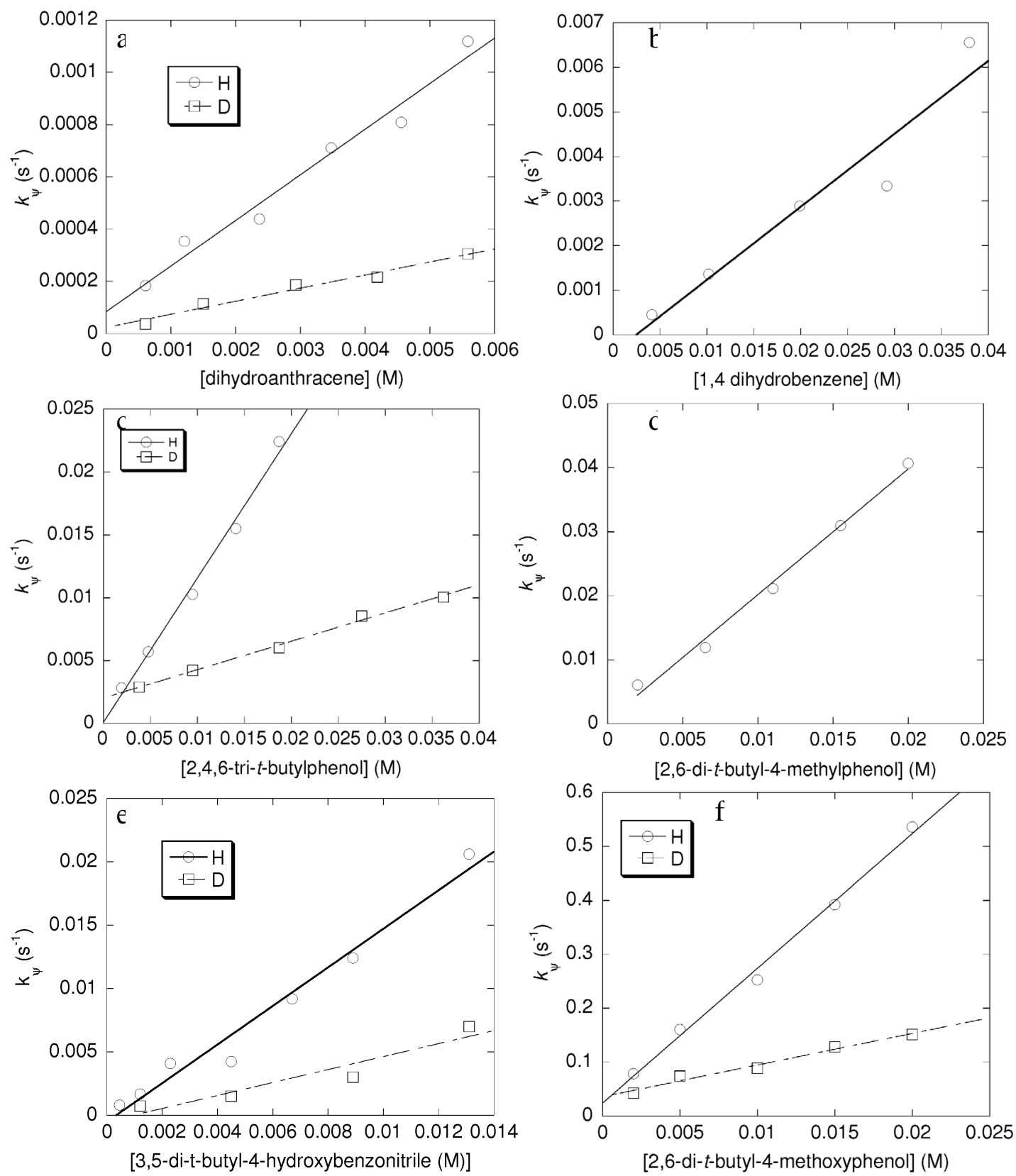

Figure S1. Plots of $k_{\psi}$ vs. [substrate] for HAT reactions. $y$-Intercepts for some deuterated phenols and 3,5-di-t-butyl-4-hydroxyacetophenone are indicative of the presence of residual water or impurity that could not be removed in the purification process. (a) [9,10-dihydroanthracene $]=0.61-5.6 \mathrm{mM}: k_{\mathrm{H}}=$ $0.175 \pm 0.015 \mathrm{M}^{-1} \mathrm{~s}^{-1}, \mathrm{R}=0.99 ; k_{\mathrm{D}}=0.049 \pm 0.005 \mathrm{M}^{-1} \mathrm{~s}^{-1}, \mathrm{R}=0.99$. (b) [1,4 dihydrobenzene $]=4.1-38$ $\mathrm{mM}: k_{\mathrm{H}}=0.16 \pm 0.03 \mathrm{M}^{-1} \mathrm{~s}^{-1}, \mathrm{R}=0.96$. (c)[2,4,6-tri-tert-butylphenol] $=1.9-19 \mathrm{mM},[2,4,6$-tri-tertbutylphenol- $d]=3.8-36 \mathrm{mM}: k_{\mathrm{H}}=1.15 \pm 0.06 \mathrm{M}^{-1} \mathrm{~s}^{-1}, \mathrm{R}=0.996 ; k_{\mathrm{D}}=0.22 \pm 0.009 \mathrm{M}^{-1} \mathrm{~s}^{-1}, \mathrm{R}=0.998$. (d) [2,6-di-tert-butyl-4-methylphenol] $=2.0-20.0 \mathrm{mM}: k_{\mathrm{H}}=2.0 \pm 0.1 \mathrm{M}^{-1} \mathrm{~s}^{-1}, \mathrm{R}=0.996$. (e) [2,6-di-tertbutyl-4-cyanophenol] $=0.46-13 \mathrm{mM}: k_{\mathrm{H}}=1.52 \pm 0.11 \mathrm{M}^{-1} \mathrm{~s}^{-1}, \mathrm{R}=0.99 ; k_{\mathrm{d}}=0.51 \pm 0.11 \mathrm{M}^{-1} \mathrm{~s}^{-1}, \mathrm{R}=$ 0.95. (f) [2,6-di-tert-butyl-4-methoxyphenol] $=2-20 \mathrm{mM} ; k_{\mathrm{H}}=25 \pm 1 \mathrm{M}^{-1} \mathrm{~s}^{-1}, \mathrm{R}=0.997 ; k_{\mathrm{d}}=5.9 \pm 0.5$ $\mathrm{M}^{-1} \mathrm{~s}^{-1}, \mathrm{R}=0.99$. 


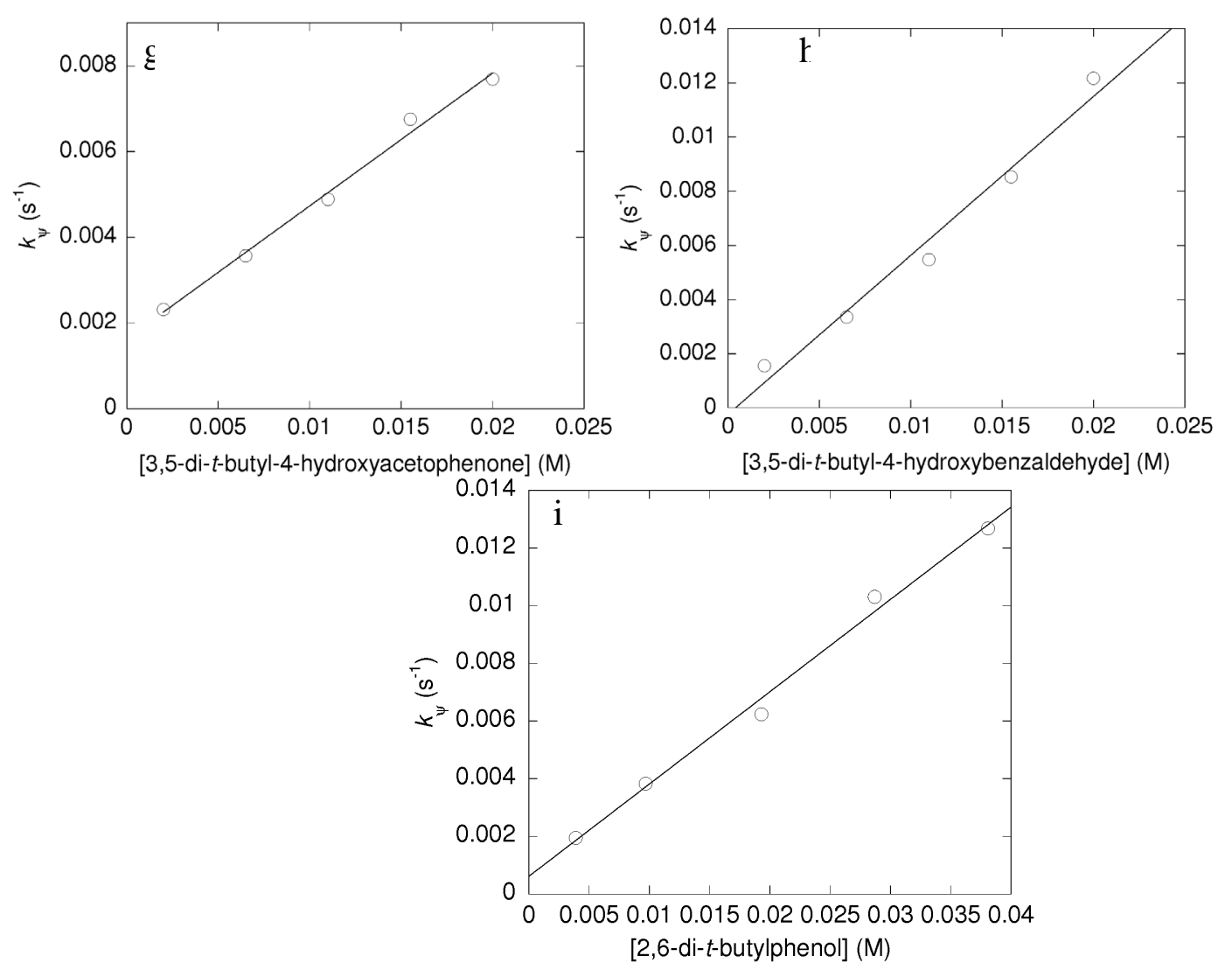

Figure S1 (continued). Plots of $k_{\psi}$ vs. [substrate] for HAT reactions. (g) [3,5-di-tert-butyl-4hydroxyacetophenone $]=2.0-20.0 \mathrm{mM}: k=0.175 \pm 0.015 \mathrm{M}^{-1} \mathrm{~s}^{-1}, \mathrm{R}=0.99 ; k_{\mathrm{D}}=0.049 \pm 0.005$ $\mathrm{M}^{-1} \mathrm{~s}^{-1}, \mathrm{R}=0.99$. (h) [3,5-di-tert-butyl-4-hydroxybenzaldehyde] $=2.0-20.0 \mathrm{mM}: k=0.59 \pm$ $0.05 \mathrm{M}^{-1} \mathrm{~s}^{-1}, \mathrm{R}=0.99$. (i)[2,6-di-tert-butylphenol] $=3.9-38 \mathrm{mM}: k=0.32 \pm 0.02 \mathrm{M}^{-1} \mathrm{~s}^{-1}, \mathrm{R}=$ 0.996 ; 
Repeat Scans on reaction of (tpfc)MnNTs with excess 2,6-di-tert-butylphenol.
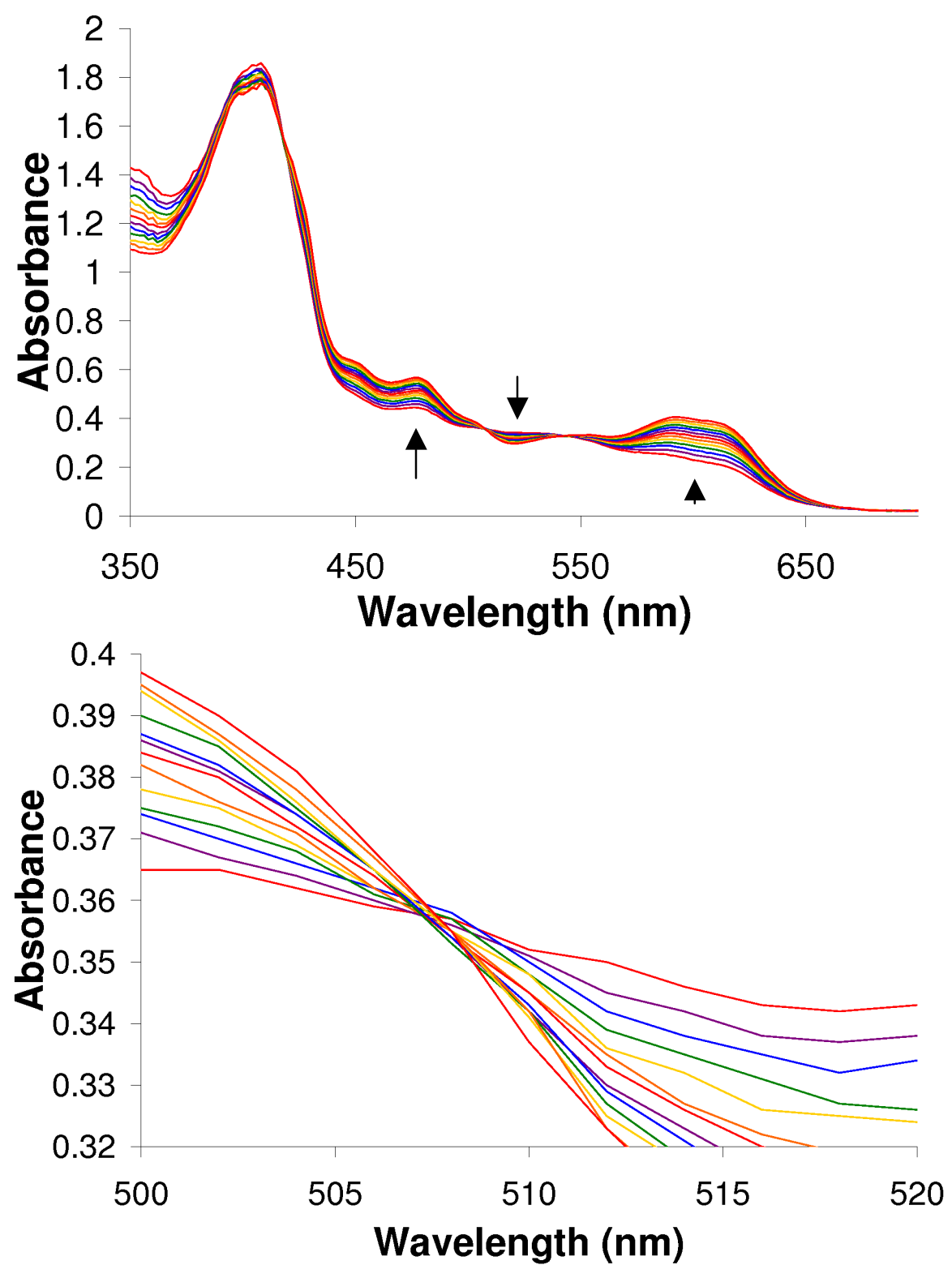

Figure S2. UV-Vis spectra showing conversion between 2 and 1 by reaction of (tpfc)MnNTs (2) with 2,6-di-tert-butylphenol. Top: Full window. Bottom: Zoomed view showing imperfect isosbestic point at $507 \mathrm{~nm}$. [2] $]_{0}=4.85 \times 10^{-5} ;\left[2,6\right.$-tert-butylphenol] $=4.85 \times 10^{-4}$. Scan interval $=1$ minute. 


\section{EPR detection of phenoxyl radicals}

In toluene under dry, anaerobic conditions, $\mathbf{2}$ was generated by stirring $1 \mathrm{mg} 1$ with $1 \mathrm{mg}$ of $2-(t-$ butylsulfonyl)( $p$-toluene-sulfonyliminoiodo)benzene to afford a dark red solution. This was added to $10 \mathrm{mg}$ of the corresponding phenol in an EPR tube. The solution was flash frozen in liquid nitrogen, and analyzed by EPR spectroscopy.

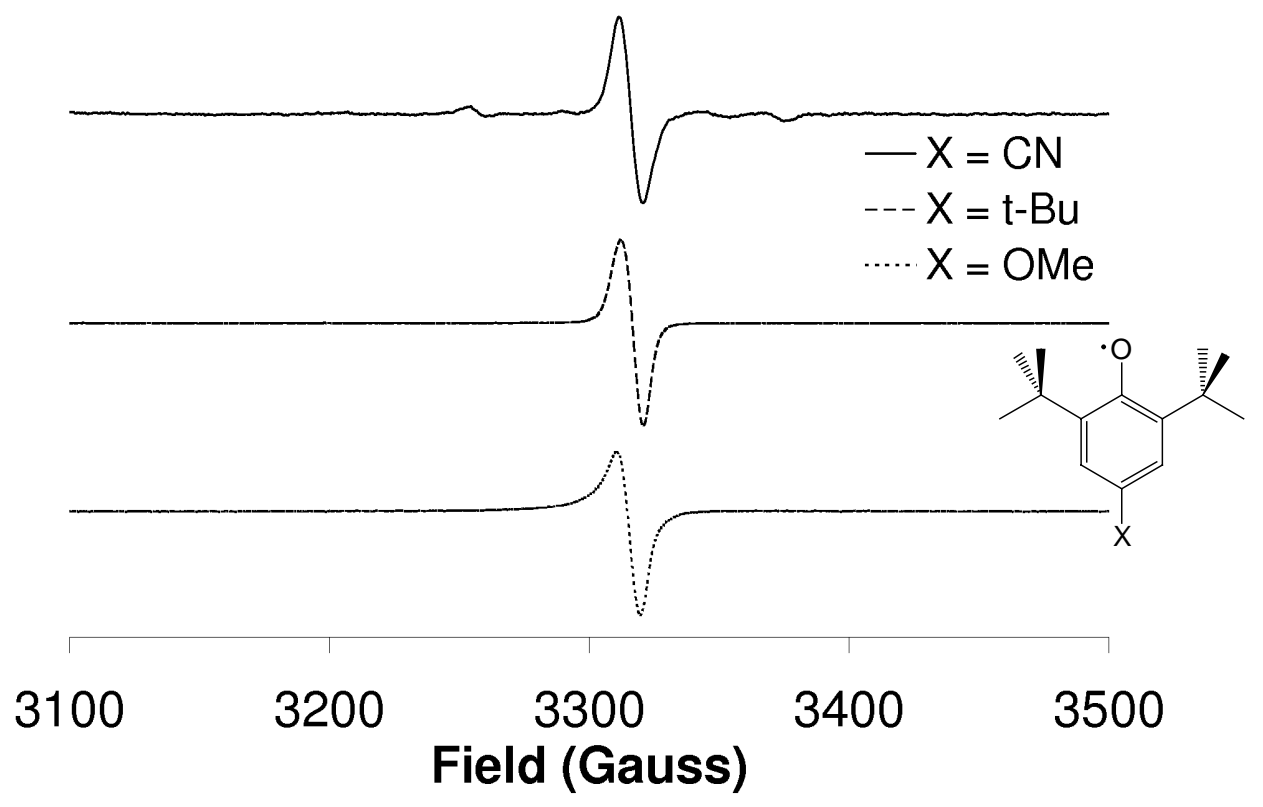

Figure S3. Phenoxyl radical signatures at $g=2.003$. $T=5 \mathrm{~K}$; Microwave Frequency $=9.30$ GHz; Mod. Freq. $=100 \mathrm{kHz}$; Mod. Amplitude $=7.182 \mathrm{G}$; for $\mathrm{X}=\mathrm{CN}$ (top), Microwave Power $=$ $1.00 \mathrm{~mW}$; for $\mathrm{X}={ }^{t} \mathrm{Bu}$ (middle), Microwave Power $=0.0100 \mathrm{~mW}$; for $\mathrm{X}=\mathrm{OMe}$ (bottom), Microwave Power $=2.00 \times 10^{-4} \mathrm{~mW}$. 


\section{Activation parameters}
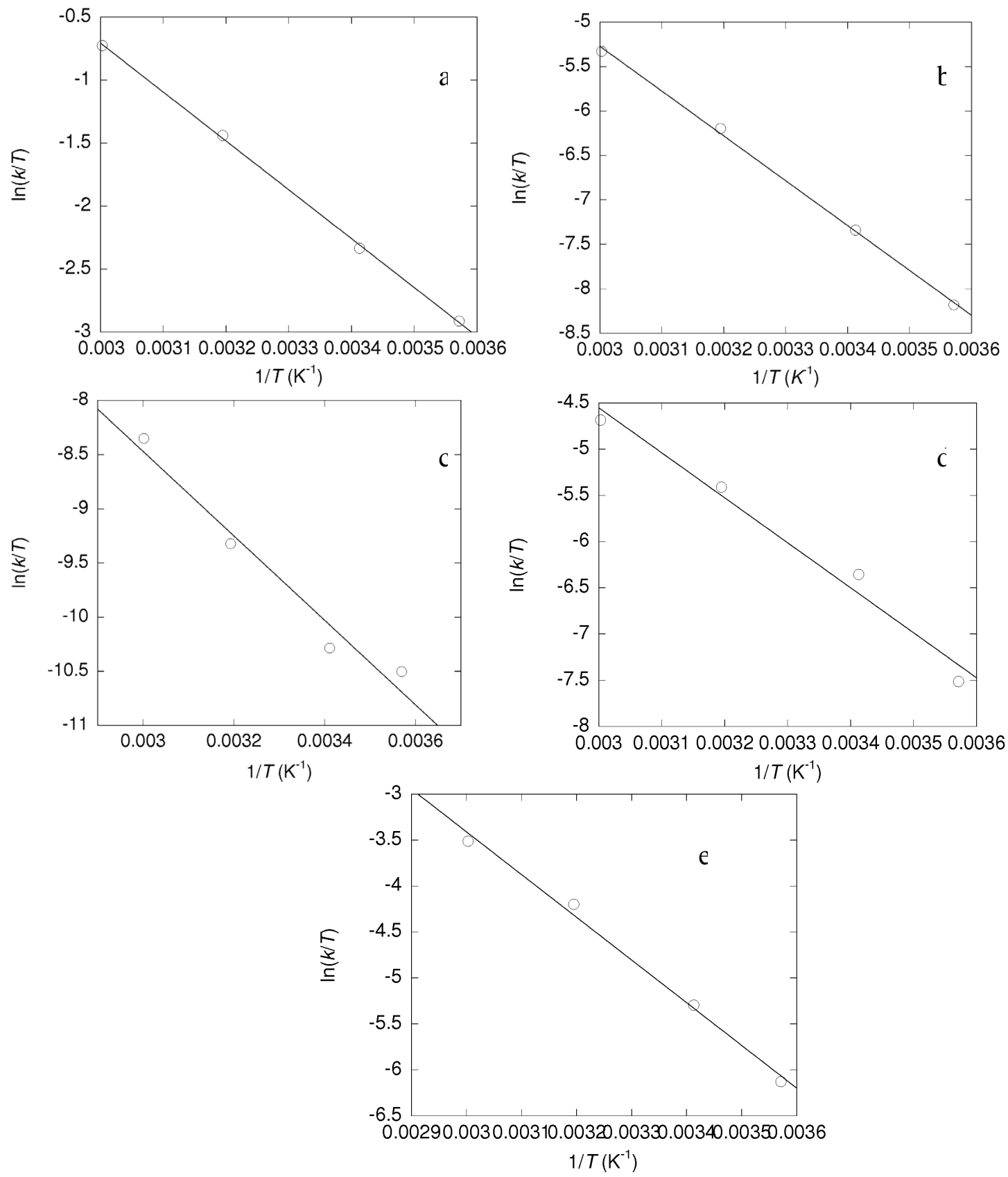

Figure S4. Determination of activation parameters for HAT from phenol substrates. Phenol was held in excess to attain pseudo-first-order conditions in the disappearance of $\mathbf{2}$. Temperature range $7-60^{\circ} \mathrm{C}$. a) [2,6-di-tert-butyl-4-methoxyphenol] $=2.48 \mathrm{mM} ; \mathrm{R}=0.99975 \mathrm{~b}$ ) [3-5-ditert-butyl-4-methylbenzaldehyde] $=21.3 \mathrm{mM} ; \mathrm{R}=0.99937$ c) [2,6-di-tert-butyl-4-cyanophenol] $=200 \mathrm{mM} ; \mathrm{R}=0.9815$ d) [2,6-di-tert-butylphenol] $=2.45 \mathrm{mM} ; \mathrm{R}=0.9891 \mathrm{e})[2,6$-di-tertbutyl-4-methylphenol] $=2.45 \mathrm{mM}$; 0.99672. Activation parameters are shown in Table 1 . 


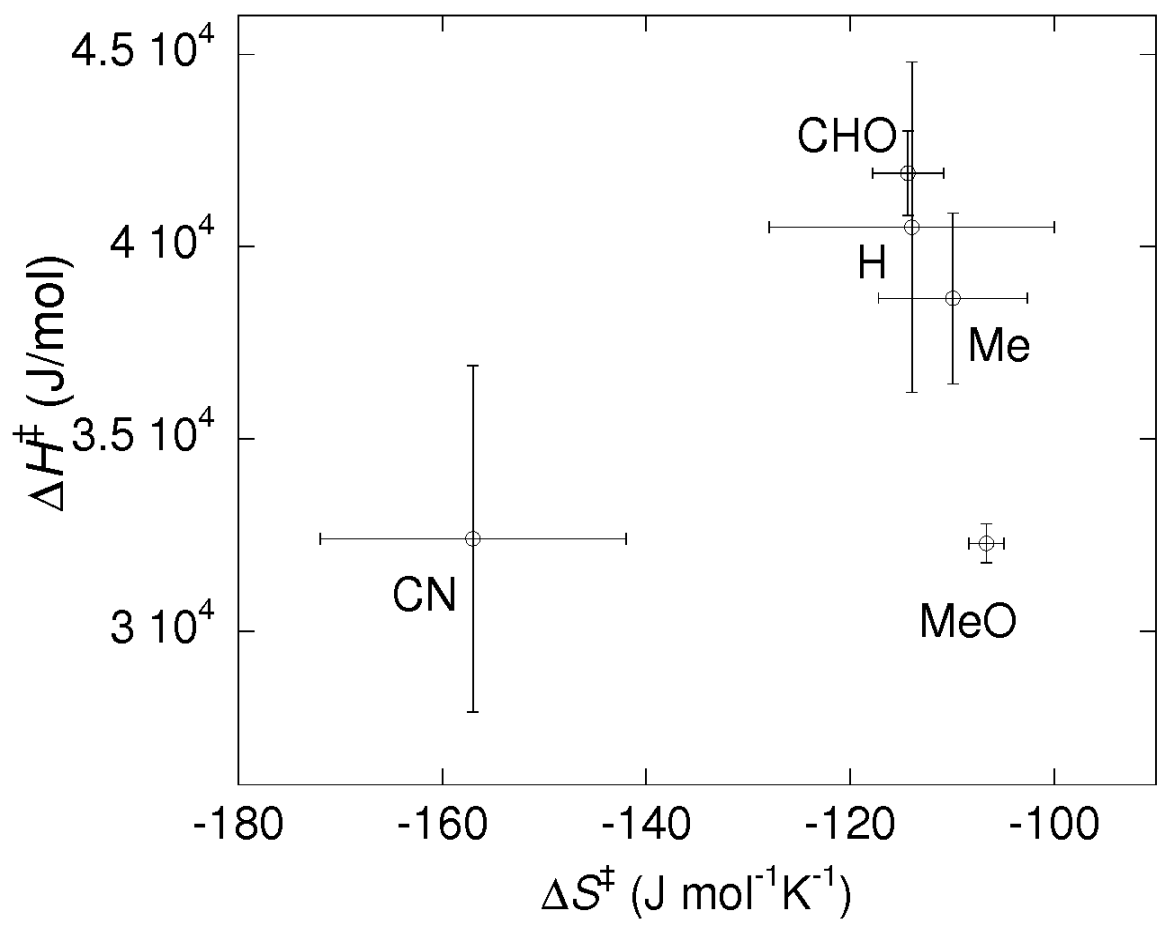

Figure S5. Isokinetic correlation plot of activation parameters in Table 1. Error bars represent 1 standard devation as determined by the linear regression fits of Fig. S4. 


\section{Mass Spectra of 4 and 5}

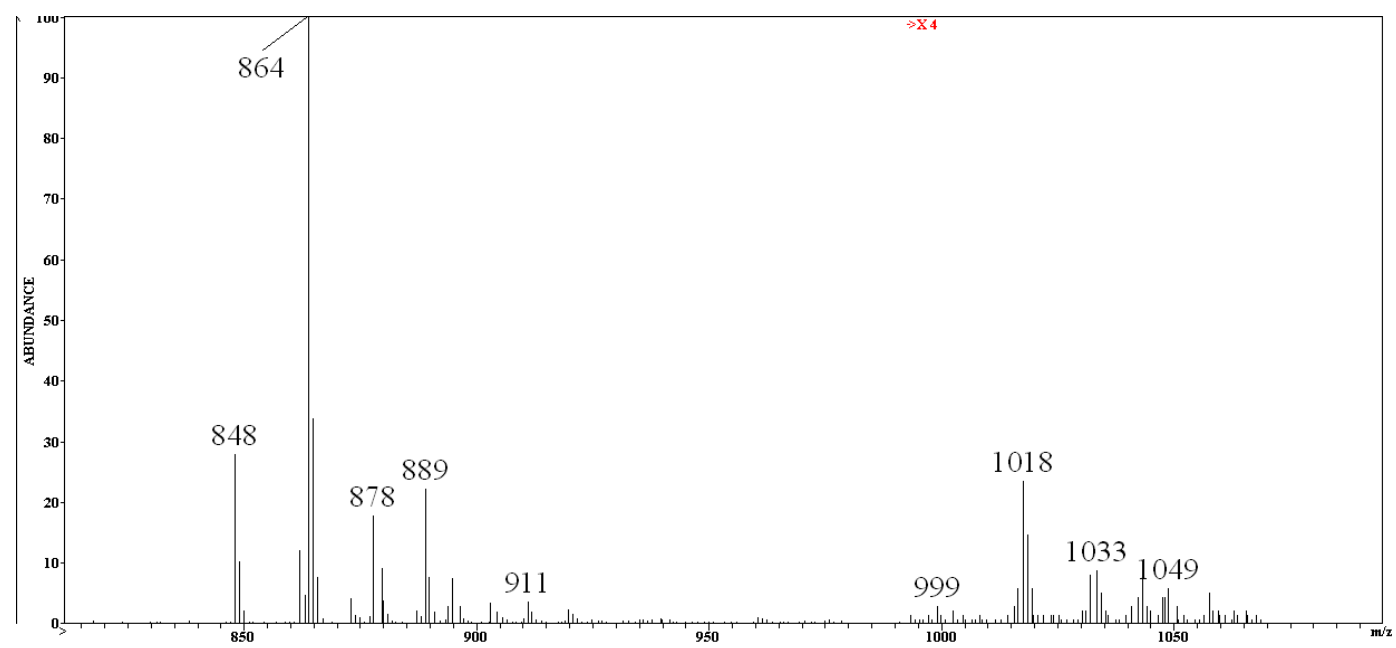

Figure S6. ESI mass spectrum of (tpfc) $\mathrm{Mn}^{\mathrm{V}}(\mathrm{NHTs})^{+}$showing parent ion peak at $\mathrm{m} / \mathrm{z}=1018$.

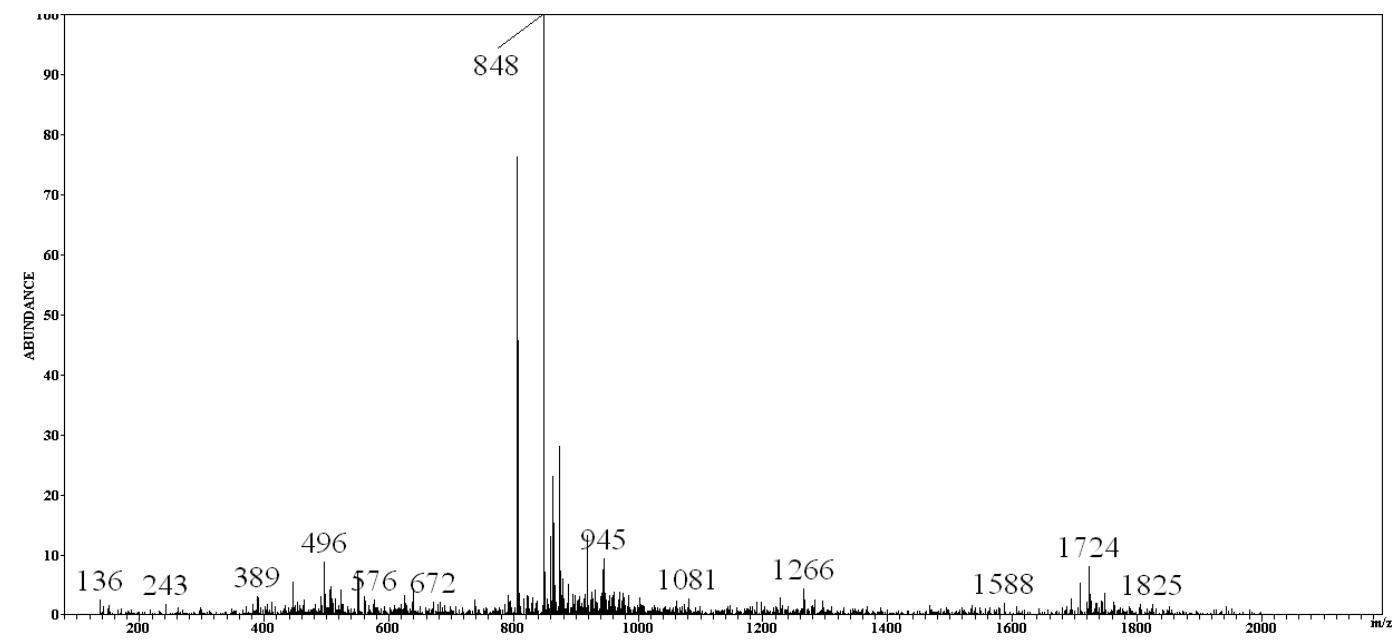

Figure S7. ESI mass spectrum of $\left[(\mathrm{tpfc}) \mathrm{Mn}^{\mathrm{IV}}\right]^{+}$showing parent ion peak at $\mathrm{m} / \mathrm{z}=848$. 